enhancement (abstraction of, say, breast cancer size, grade and nodal status from pathology reports notified to the cancer registry or linking cancer registry to inpatient statistics data) or by methods for which there are already ample precedents (a clinical cancer data collection, a patterns of care survey, or a survey of patients themselves).

The papers in this special cancer issue show how easy it is. Helen Moore and colleagues outline a structured and rigorous way of arriving at a parsimonious and simple set of indicators for measuring the performance of clinical services for melanoma control. Sounds difficult? Not really. It has to be done with care, but it required only two meetings of about two hours with the expert advisory group to reach a firm consensus on what should be measured. That beats endless meetings debating 'what data we should collect' and risking an uncollectable, unmanageable and often unusable data collection in the end. In other papers, Churches and Lim show what can be achieved in measuring breast cancer services through the linkage of the cancer registry and inpatient statistics collections and Kricker shows what can be done as well when data from pathology reports are added; Taylor and colleagues report the results of linkage of BreastScreen data with cancer registry data to produce interval cancer rates, the key measure of mammographic screening performance; and Macansh shows what the Pap Test Register has to offer on performance indicators for cervical screening. In an earlier issue (NSW Public Health Bulletin 2001; 12 (1): 2-6), Moore and colleagues illustrated the value of the NSW Health Survey in measuring risk factors for cancer.

To move from where we are now in measuring the performance of cancer services in NSW to where, ideally, we should be still requires the introduction of standardised clinical cancer information systems in all the main cancer treatment centres in the State, which are linked to the NSW Central Cancer Registry. Requiring less development, but equally as important, in measuring and improving the performance of cancer services in NSW are:

- a regular program of surveys of cancer care 'consumers'

- a planned approach to analysis of linked cancer registry and inpatient statistics data sets

- a continued program of enhanced analysis of cancer registry data

- patterns of care surveys 'to fill the gaps' in population coverage

- continuation of full exploitation of the richness of the Cancer Registry, Pap Test Register and BreastScreen data sets. $\mathbb{W}$

\title{
WORKING OUT WHAT TO MEASURE: MELANOMA SERVICES
}

Helen Moore, Cait Lonie, * and Hanna Noworytko Epidemiology and Surveillance Branch NSW Department of Health

\section{Bruce Armstrong}

Research and Registers Division NSW Cancer Council

This article reports on a process to identify a priority set of indicators to measure the performance of services for melanoma, outlined in the Australian Cancer Network's Guidelines for the Management of Cutaneous Melanoma published in June 1997. ${ }^{1}$ Melanoma is a major cause of morbidity in NSW. In 1998 , the year for which there is the most recently available data, it was the fourth most common cancer diagnosed in NSW residents, with 1,565 cases diagnosed in males and 1,119 in females; ${ }^{2}$ accounting for 362 deaths, three per cent of all deaths caused by cancer. Melanoma was the most common cancer in males and females aged 15-39 years in 1998.

The importance of reducing this morbidity and associated mortality was recognised in 1994 by the Cancer Expert Working Group when they set goals and targets for NSW to reduce the incidence of, and mortality due to, melanoma. ${ }^{3}$ To assist in achieving these goals, a health

* Currently psychiatric registrar, Chatswood Community Mental Health Centre. outcomes approach was applied to melanoma to identify areas for intervention across the continuum of care from prevention through to treatment and palliation or rehabilitation. ${ }^{4}$

\section{HEALTH OUTCOMES APPROACHTO REDUCE MORBIDITY AND MORTALITY FROM MELANOMA IN NSW}

Specific opportunities to reduce morbidity and mortality due to melanoma are presented in Figure 1. In general, the intervention points that will produce health gains for the population and for people with melanoma are:

- preventing the development of melanoma by reducing exposure to known causal agents: for example, high intensity intermittent exposure to UV radiation;

- detecting and diagnosing cutaneous melanoma as early as possible;

- giving appropriate psychosocial support to patients with suspected or confirmed melanoma and their families;

- managing primary operable melanoma in accordance with international best practice;

- managing advanced melanoma in accordance with international best practice;

- providing best practice palliative care to those who will die from melanoma. 


\section{FIGURE 1}

MODIFIABLE RISK FACTORS, INTERVENTION POINTS, AND INTERVENTIONS TO REDUCE THE INCIDENCE OF, AND MORBIDITY AND MORTALITY FROM, CUTANEOUS MELANOMA

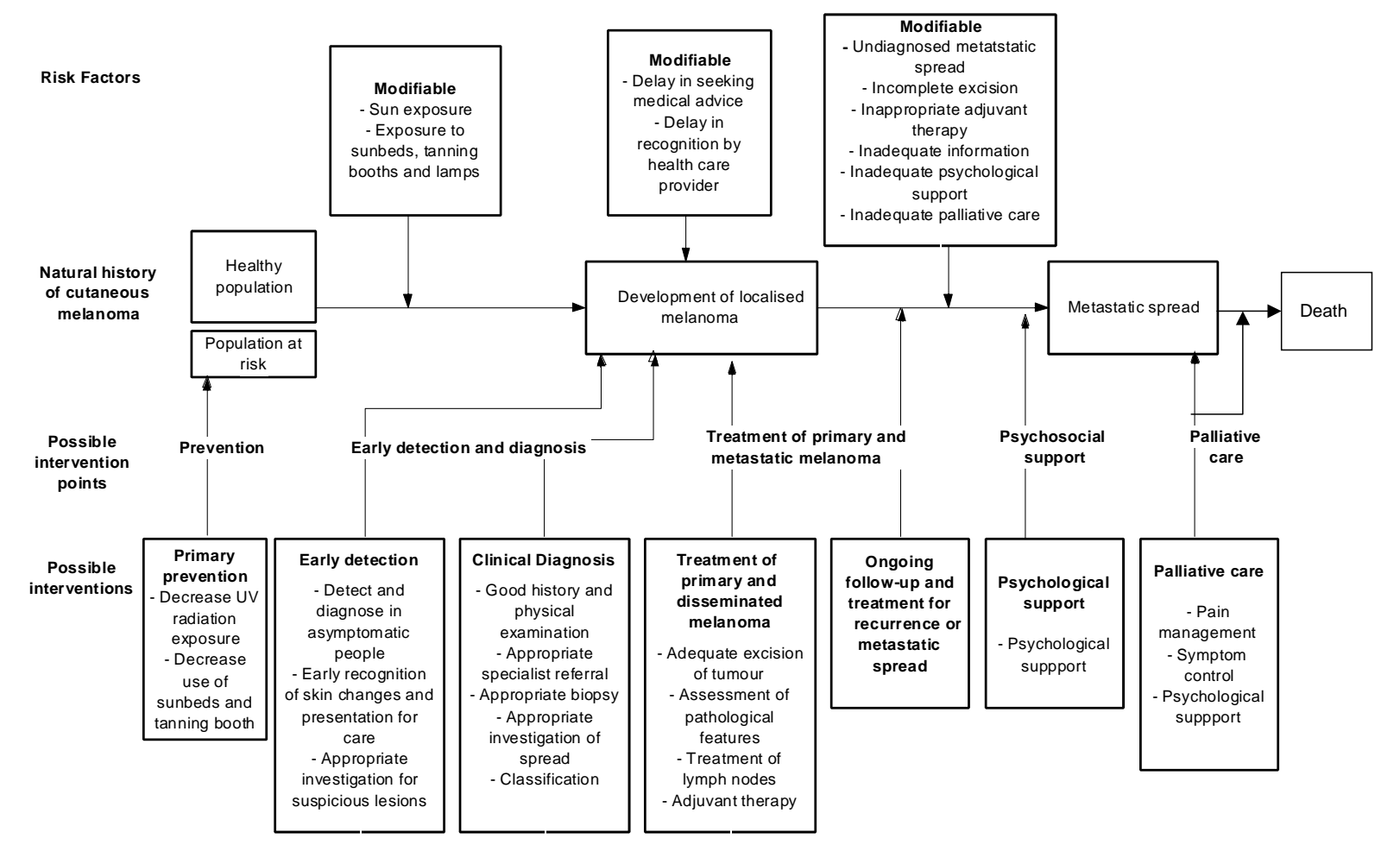

The NSW Department of Health, in conjunction with the NSW Cancer Council, has implemented a range of interventions to prevent skin cancers under a series of skin cancer control strategic plans. ${ }^{5,6}$ Recommendations on best practice in relation to the clinical intervention points are outlined in the Australian Cancer Network's Guidelines for the Management of Cutaneous Melanoma published in June 1997. ${ }^{1}$ The National Health and Medical Research Council endorsed these Guidelines in December, 1999. ${ }^{7}$ The Guidelines provide advice to a range of service providers on the principles of melanoma management based on the best evidence currently available. They cover the spectrum of care from prevention through to early detection and diagnosis, management and palliation.

Research into guideline development has shown that the way guidelines are developed, implemented and monitored determines how effective they are in changing clinical practice. ${ }^{8}$ A critical aspect of effective guideline implementation is the integration of the guidelines into a quality improvement process. This process entails monitoring the effect of the guidelines on practice, feedback of information collected and readjustment of implementation where necessary to improve practice and ultimately outcomes.

Efficient monitoring requires the development of an efficient set of performance indicators. The development, and particularly the implementation, of performance indicators is a time consuming and expensive process. The cost-effectiveness of the information proposed for collection must be considered, and the indicators should be prioritised according to the expected benefits of their use in quality improvement. The priority set of indicators to measure the performance of services for melanoma, outlined in the Australian Cancer Network's Guidelines for the Management of Cutaneous Melanoma, may form the basis for the development of a melanoma module for Clinical Cancer Registries, which are currently being implemented in NSW (see article by Noworytko et al. in the February 2001 issue of the NSW Public Health Bulletin Volume 12, Number 2).

\section{STEPS IN DEVELOPING A PRIORITY SET OF INDICATORS FOR MELANOMA CLINICAL SERVICES}

The model used to develop an expanded set of indicators for monitoring melanoma clinical services was that proposed by the Quality and Outcomes Monitoring Working Party for the Optimising Cancer Management Initiative. ${ }^{9}$ This process involved preparing a set of objectives for care and associated interventions; developing indicators for each objective; and assessing the benefits and costs of the indicators. Indicators were ranked in order of priority and the ranked list was further refined. The details and results of this process are as follows:

\section{Preparing a set of general objectives and associated intervention types}

These objectives described the outcomes to which melanoma services are directed and the interventions required, on either theoretical or empirical grounds, to 


\section{TABLE 1}

\section{MELANOMA CLINICAL INDICATOR FRAMEWORK: OBJECTIVES AND INTERVENTIONS FOR REDUCING}

MORBIDITY AND MORTALITY DUE TO CUTANEOUS MELANOMA

\begin{tabular}{|c|c|}
\hline Objective & Description \\
\hline Objective 1 & $\begin{array}{l}\text { Reduce the incidence of cutaneous melanoma } \\
\text { No interventions were specified, as the focus of the indicator identification process was primarily clinical. }\end{array}$ \\
\hline $\begin{array}{c}\text { Objective } 2 \\
\text { Interventions } \\
2.1 \\
2.2 \\
2.3 \\
2.4 \\
2.5\end{array}$ & $\begin{array}{l}\text { Detect and diagnose cutaneous melanoma at the earliest possible stage } \\
\text { Detect and diagnose melanoma in asymptomatic people; } \\
\text { Detect and diagnose melanoma in symptomatic people at the earliest possible stage; } \\
\text { Minimise the excision of benign melanocytic naevi; } \\
\text { Investigate symptoms and signs of melanoma in accordance with international best practice; } \\
\text { Report on pathology findings in accordance with international best practice. }\end{array}$ \\
\hline $\begin{array}{c}\text { Objective } 3 \\
\text { Interventions } \\
3.1 \\
3.2 \\
3.3 \\
3.4 \\
3.5\end{array}$ & $\begin{array}{l}\text { To achieve and maintain optimal psychosocial adaptation in people with suspected or confirmed } \\
\text { melanoma and their families } \\
\text { Promote optimism in patients in regard to their management and quality of life; } \\
\text { Give psychosocial support to people who have been diagnosed with melanoma and their families; } \\
\text { Inform people who have been diagnosed with melanoma of their diagnosis, the prognosis of their cancer, } \\
\text { the proposed treatment and likely outcomes; } \\
\text { Involve people who have been diagnosed with melanoma in making decisions regarding treatment to the } \\
\text { level they want to be involved; } \\
\text { Diagnose and treat psychological morbidity in accordance with best practice principles. }\end{array}$ \\
\hline $\begin{array}{r}\text { Objective } 4 \\
\text { Interventions } \\
4.1 \\
4.2 \\
4.3\end{array}$ & $\begin{array}{l}\text { Manage primary operable melanoma in accordance with international best practice } \\
\text { Ensure management decisions are based on pathological features; } \\
\text { Ensure management of specific types and sites of melanoma is in accordance with international best } \\
\text { practice; } \\
\text { Ensure patients with melanoma have appropriate follow-up organised. }\end{array}$ \\
\hline $\begin{array}{c}\text { Objective } 5 \\
\text { Interventions } \\
\qquad \begin{array}{c}5.1 \\
5.2 \\
5.3\end{array}\end{array}$ & $\begin{array}{l}\text { Manage locoregionally advanced melanoma in accordance with best practice principles } \\
\text { Ensure management decisions are based on pathological features; } \\
\text { Ensure management of lymph nodes is in accordance with international best practice; } \\
\text { Ensure patients with locoregionally advanced melanoma have appropriate follow-up organised. }\end{array}$ \\
\hline $\begin{array}{c}\text { Objective } 6 \\
\text { Interventions } \\
\qquad \begin{array}{c}6.1 \\
6.2 \\
6.3 \\
6.4\end{array}\end{array}$ & $\begin{array}{l}\text { Manage patients with disseminated melanoma in accordance with international best practice } \\
\text { Ensure access to appropriate surgical, radiotherapeutic and chemotherapeutic services for patients with } \\
\text { disseminated melanoma in whom anti-tumour therapy is judged to offer potential benefit in quality survival; } \\
\text { Ensure access to palliative care for people with disseminated melanoma and their carers when quality of life } \\
\text { (including physical, social, emotional, spiritual or financial aspects), is impaired by the disease; } \\
\text { Ensure palliative care services are of the best quality and are in accordance with international best practice; } \\
\text { Improve multidisciplinary management of patients with advanced melanoma. }\end{array}$ \\
\hline
\end{tabular}

achieve them. Table 1 summarises the objectives and interventions used as a framework to guide development of the performance indicators.

\section{Developing indicators for each objective}

These were outcome indicators, or measures of the outcome described by the indicator (for example, death or healthrelated quality of life); risk indicators (determinants of the outcome); or process indicators (measures of success in implementing an intervention for which there is evidence of effectiveness in achieving the measures of outcome).

The final list contained 71 indicators that were further defined by the population and data items required for each indicator and the methods for collecting the necessary data.

\section{Assessing the benefits and costs of the proposed} indicators

This involved reviewing the available literature to assess the effectiveness of the underlying interventions and hence the quality of the evidence for the process indicators.
The potential data sources for each indicator were identified as a proxy for the cost of data collection.

Of the 71 indicators, 13 were from sources which are currently available; 29 were from current sources that required further work to provide the data in a useable form (such as linking data from the NSW Central Cancer Registry with the Inpatients Statistics Collection); and 29 were from sources which required extensive developmental work (such as Clinical Cancer Registries).

When attempting to define the costs and benefits of each indicator, it became clear that there was little information available to make these estimates rigorously. Therefore opinions of an expert advisory group, the NSW Melanoma Clinical Indicators Working Party, were sought to assess the information on effectiveness and costs and provide an expert opinion on where the greatest health gain would be achieved at the least cost by monitoring the indicator. Most of the members of the Working Party were directly involved in the clinical management of melanoma and 
represented a range of oncology specialties, psychiatry, pathology and epidemiology.

\section{Ranking the indicators}

To determine an order of priority for the indicators, each member of the NSW Melanoma Clinical Indicators Working Party ranked the list of indicators using the criteria listed in Table 2. Each Working Party member then scored each indicator on a 1 to 4 scale with the following importance:

- use of the indicator would be highly cost-effective in improving melanoma outcomes;

- use of the indicator would be moderately costeffective in improving melanoma outcomes;

- use of the indicator would be weakly cost-effective in improving melanoma outcomes;

- use of the indicator would not be cost-effective in improving melanoma outcomes.

Fourteen of the 17 members of the Working Party participated in the exercise. The fourteen scores for each indicator were summed and indicators ranked in ascending order of the total scores.

\section{Identifying priority indicators}

The Working Party members reviewed the results of the ranking exercise, agreed on the order by priority of the list of indicators, and selected a minimum set of cost-effective indicators and associated data collection

\section{TABLE 2}

\section{MELANOMA CLINICAL INDICATORS: CRITERIA FOR RATING EACH INDICATOR}

Each member of the Melanoma Clinical Indicators Working Party was asked to use the following criteria to evaluate the cost-effectiveness of each indicator:

\section{The Intervention}

- how effective the intervention underlying the indicator is in improving health or quality of life;

- the strength of evidence that the intervention does produce a beneficial outcome;

- the extent of current variation in clinical practice from best practice, as measured by the indicator.

\section{The Indicator}

- the quality of the indicator including validity, measurement accuracy, timeliness in relation to events measured, responsiveness to change in practice and stability of quality over time;

- the potential usefulness of the indicator in moving current practice towards best practice;

- the feasibility of measuring the indicator;

- the cost of measuring the indicator.

\section{Equity}

Can the indicator be used to measure and promote equity of service or outcome in relation to characteristics such as socioeconomic status, place of residence, indigenous status and country of birth?

A broad concept of equity is intended here, not just access to services. It should include:

- inequitable distribution of knowledge about health and health services;

- inequitable distribution of health and ill health;

- inequitable quality or outcome of health care. vehicles. This part of the process was largely based on the expert opinion of Working Party members.

A cut-off line was arbitrarily drawn in the indicator list. This included 30 indicators 'above the line', consisting of the top ranked 27 indicators and three indicators not ranked as highly, but for which data were currently available.

From this starting point, all Working Party members discussed which indicators above the cut-off line should be removed and which indicators from below the cut-off line should be included. The arguments for including an indicator that was not ranked in the top 30 were that the information provided by the indicator was unique, or there was relatively strong evidence of health gain or it was cost-effective to collect.

This process resulted in the identification of 43 priority indicators from the original list of 71 candidate indicators. These are priority indicators are listed in Table 3 with availability of their data source.

\section{CONCLUSION}

This paper describes a process that aimed at defining a minimum set of indicators to monitor key aspects of services for melanoma in NSW. These indicators are relevant to clinicians and clinical practice; related to the most important aspects of clinical practice; could potentially assist with changing practice through feedback; and allowed the monitoring of variations in practice that may affect patient outcomes. When clinical cancer information systems begin to operate, it is hoped that subsets of these indicators may be measured-and reported on regularly - both statewide and at major cancer treatment centres.

In summary, the process involved systematically developing an extended list of indicators measuring the desired outcomes of care for melanoma, either directly or indirectly, as processes of care known or thought to be effective in producing those outcomes. These indicators were placed in order of priority through consensus of a group of experts informed by evidence of the effectiveness of interventions targeted by the indicators and the likely costs of measuring them. The process required the participation of a multidisciplinary group that met twice and was responsible for individually assessing the extended list of indicators and collectively agreeing on priority indicators.

The process was systematic, explicit and documented; it focused on areas of greatest health gain; it was evidencebased; and it involved stakeholders. It allows for the cost of implementing data collection systems to be more reliably estimated, as it assists in specifying the system outputs and functions. It also assists in generating evidence on the effectiveness of particular clinical interventions, as it provides information about the relative importance, clinically, of monitoring the outcomes of these interventions and it highlights the most important information gaps. 
TABLE 3

LIST OF MELANOMA CLINICAL INDICATORS IN PRIORITY ORDER

Indicator

Data source

availability* $^{*}$

1. Age, sex and site specific, and age-adjusted incidence of melanoma.

2. Proportion of newly diagnosed melanomas that are in-situ, $.0 .75 \mathrm{~mm}, 0.76-1.50 \mathrm{~mm}, 1.51-3.00 \mathrm{~mm}$ and $>3 \mathrm{~mm}$ thick, and median Breslow thickness.

3. Proportion of people with newly diagnosed melanomas with localised disease at diagnosis.

4. Age and sex specific and age-adjusted mortality from melanoma.

5. Proportion of people at high risk of melanoma who are participating in a skin surveillance program.

6. Proportion of high risk individuals identified as such by their self, doctor or other family member.

7. Proportion of people at high risk of melanoma who are undertaking monthly skin self-examination.

8. Proportion of adults who have been asked if they have a family history of melanoma.

9. Proportion of people referred to cancer genetic services for melanoma, with a genuinely higher familial risk.

10. Proportion of genetic tests performed that detect high-risk mutations for family history of melanoma.

11. Stage-specific and stage-adjusted survival after diagnosis of melanoma.

12. Proportion of patients with a non-facial melanoma less than $1 \mathrm{~mm}$ thick who have any type of closure other than a primary closure (Including those with excision margins greater than $1 \mathrm{~cm}$ and which result in skin grafts or flaps).

13. Proportion of people with locoregionally advanced or disseminated melanoma who are referred for treatment of the disease to a centre specialising in the treatment of melanoma, and where multidisciplinary care is available.

14. Proportion of patients with melanomas 1 to $3 \mathrm{~mm}$ thick on the trunk or above the elbow or knee who have a skin graft or flap repair to close the wound.

15. Proportion of patients with disseminated melanoma who stay 25 days or more in hospital in their last 100 days of life.

16. Proportion of patients with mucosal melanoma referred to a speciality clinic or clinician.

17. Proportion of melanoma specimens that are diagnosed with a shave or punch biopsy.

18. Proportion of histopathological reports on melanoma that include information on tumour thickness, Clark level and margins.

19. Proportion of melanomas submitted for histopathological diagnosis by total excision with margin of $2 \mathrm{~mm}$ or more and to the upper layer of fat

20. Age specific rates of histopathological diagnosis of melanocytic naevi to that of melanomas.

21. Proportion of pathology request forms that include complete information on: patient identification; clinical details of the lesion (size, site, history of lesion etc); history of previous melanoma; and provisional clinical diagnosis.

22. Proportion of people with newly diagnosed melanoma who sought medical advice within four weeks of noticing skin changes.

23. Proportion of people with newly diagnosed melanoma who had diagnosis made within four weeks of first reporting relevant symptoms to a doctor.

24. Proportion of people diagnosed with melanoma who report not receiving enough information on melanoma, their diagnosis and prognosis, the treatments available and their likely outcomes.

25. Proportion of patients who are optimistic that they and their disease will be managed in the most effective and caring way.

26. Proportion of patients diagnosed with clinical levels of anxiety, depression or abnormal illness behaviour who are referred to a counselling service.

27. Proportion of patients who are optimistic that they will be able to maintain a good quality of life.

28. Proportion of people who report having unmet needs in relation to their family and carer's fears, coping and access to support.

29. Quality of life of people diagnosed with melanoma, and their carers by disease status at the time of measurement.

30. Proportion of patients with an unmet need for help with physical symptoms or emotional stress due to their melanoma.

31. Proportion of patients with melanoma who have undetected clinical levels of anxiety, depression or abnormal illness behaviour.

32. Proportion of patients who died from melanoma who were offered appropriate 24 hour nursing or medical services for palliative care in the home.

33. Proportion of primary melanomas correctly excised or re-excised with margins indicated by tumour thickness measurement and type (including desmoplastic melanoma with neurotropism who have excision margins $1 \mathrm{~cm}$ greater than the usual margins).

34. Proportion of patients with invasive melanoma with the margin of excision between $1 \mathrm{~cm}$ and $3 \mathrm{~cm}$.

35. Proportion of patients with locoregionally advanced or disseminated melanoma who have multi-disciplinary care involving a surgeon, medical oncologist, radiation oncologist and palliative care specialist.

36. Proportion of people with disseminated melanoma who have a timely referral to a palliative care service.

37. Proportion of people in whom therapeutic node dissection was carried out by a surgeon with specific training in this procedure.

38. Proportion of people with suspicious lymph nodes who have fine needle aspiration of the suspicious nodes.

39. Proportion of patients who have a node dissection within two weeks of detection of nodal metastases.

40. Proportion of patients with melanoma less that $2 \mathrm{~mm}$ thick who have CT, MRI or PET scans.

41. Proportion of patients with melanoma greater than or equal to $1 \mathrm{~mm}$ thick who are included in an approved clinical trial.

42. Proportion of primary health care workers treating a patient with locoregionally advanced or disseminated melanoma who feel they have not been given adequate information on the specialist's management of the patient.

43. Proportion of GPs who use surface microscopy when examining suspicious pigmented lesions.

\footnotetext{
* Data source availability: $\quad 1=$ Currently available;

2 = Existing source but more work required;

$3=$ Extensive developmental work required.
} 
Members of the Working Party contributed about six hours each, including time spent on the ranking exercise and in meetings. Preparatory work by a project officer and manager was time consuming and included the drafting of the framework and indicators, a discussion paper, and preparing and analysing the results of the ranking exercise. Despite efforts to minimise the time spent by the clinical experts, three of the 17 Working Party members did not participate in the ranking exercise.

Delays in developing clinical information systems to support indicators may undermine the processes of developing minimum data sets to monitor the quality and outcomes of patient care. Recently, health information initiatives have been given a fresh impetus by the recommendations of the NSW Health Council, ${ }^{10}$ and the NSW Government's Action Plan for Health. ${ }^{11}$ Consequently, the time between the development of priority sets of indicators and availability of data should be reduced.

We think that the benefits of following this process of developing indicators, if realised, would justify the costs. The process provides an assurance from the data users about what should and could be measured. Therefore, we think that it ensures that resources spent on collecting data are spent giving the best possible information about the quality of services.

\section{ACKNOWLEDGEMENTS}

The authors wish to thank the remaining members of the NSW Melanoma Clinical Indicators Working Party: Professor Bruce Armstrong (Chair), Professor Alan Coates, Ms Sue Collins, Dr Neil Cooney, Dr Kerry Crotty, Professor Stewart Dunn, Dr Afaf Girgis, Professor Peter
Hersey, Dr Robyn Jordan, Professor Bill McCarthy, Dr Scott Menzies, Dr Graham Mann, Dr Bob Sillar, Dr Graeme Stevens.

\section{REFERENCES}

1. Australian Cancer Network. Guidelines for the Management of Cutaneous Melanoma. Sydney: Australian Cancer Society, June 1997.

2. Coates M, Tracey E. Cancer in New South Wales. Incidence and mortality 1998. Sydney: NSW Cancer Council, April 2001.

3. NSW Cancer Expert Working Group. Cancer Control-NSW Goals and Targets. Sydney: NSW Department of Health, August 1995.

4. Williamson M, Lonie C, Colagiuri R et al. A framework for applying a health outcomes approach. NSW Public Health Bulletin 1995; 6: 102-106.

5. Nathan S, Gaffney D. Skin cancer control in New South Wales-Health promotion Strategic Plan 1995-2000. Sydney: NSW Cancer Council and NSW Department of Health, 1995.

6. NSW Cancer Council and NSW Department of Health. Skin cancer control strategic plan 2000-2004. Sydney: NSW Cancer Council and NSW Department of Health, 2000.

7. National Health and Medical Research Council. Clinical Practice Guidelines-The management of cutaneous melanoma. Canberra: NHMRC, 1999.

8. Grimshaw $\mathbf{J}$ et al. Developing and implementing clinical practice guidelines. Quality in Health Care, 1995; 4: 55-64.

9. Quality and Outcomes Monitoring Working Party. A proposed method for identifying performance indicators for cancer services. Sydney: NSW Department of Health, 1997.

10. NSW Health Council. Report of the NSW Health Council. Sydney: NSW Department of Health, March 2000.

11. NSW Department of Health. NSW Government's Action Plan for Health-Bulletin Number 1. Sydney: NSW Department of Health, May 2000. it:

\section{THE ROLE OF THE NSW PAP TEST REGISTER IN MONITORING THE CERVICAL SCREENING PROCESS IN NSW}

\section{Sacha Macansh \\ Manager, NSW Pap Test Register \\ NSW Cancer Council}

In 1993, the Steering Group on Quality Assurance in Screening for the Prevention of Cancer of the Cervix recognised that cervical cytology registers were uniquely placed to provide comprehensive information that could be used to monitor and improve the quality of cervical screening. ${ }^{1}$ This article describes the NSW Pap Test Register, and how the data that it collects is used to monitor the performance of the NSW Cervical Screening Program. The register was established in 1996 as a central, comprehensive and confidential database of Pap test and cervical histology results for NSW women. It has a number of important functions including the collation of information that can be used to measure, monitor and improve the cervical screening process.
The Register is managed by the NSW Cancer Council and is an integral part of the NSW Cervical Screening Program. It is jointly funded by the NSW Department of Health and the Commonwealth Department of Health and Aged Care. As part of the NSW Cervical Screening Program, the Register aims to reduce the incidence of and mortality from cervical cancer by increasing participation in and improving the performance of cervical screening. The Register contributes to this aim by providing complete, accurate and timely data which can be used to measure key areas of the Program's performance.

\section{REGISTER DATA}

The Public Health Act 1991 determines that pathology laboratories must inform the NSW Pap Test Register of the results of all cervical cancer tests, Pap tests and cervical histology for NSW women. Demographic data for all 